\title{
Evaluation of antioxidant and nitric oxide inhibitory activities of selected Malaysian medicinal plants
}

\begin{abstract}
Methanol extracts of seven Malaysian medicinal plants were screened for antioxidant and nitric oxide inhibitory activities. Antioxidant activity was measured by using FTC, TBA and DPPH free radical scavenging methods and Griess assay was used for the measurement of nitric oxide inhibition in lipopolysaccharide (LPS) and interferon-כ (IFN-כ)-treated RAW 264.7 cells. All the extracts showed strong antioxidant activity comparable to or higher than that of Ŭ-tocopherol, BHT and quercetin in FTC and TBA methods. The extracts from Leea indica and Spermacoce articularis showed strong DPPH free radical scavenging activity comparable with quercetin, BHT and Vit C. Spermacoce exilis showed only moderate activity but other species were weak as compared to the standards. In the Griess assay Lasianthus oblongus, Chasalia chartacea, Hedyotis verticillata, Spermacoce articularis and Leea indica showed strong inhibitory activity on nitric oxide production in LPS and IFN-כinduced RAW 264.7 cells. Extracts from Psychotria rostrata and Spermacoce exilis also inhibited NO production but this was due to their cytotoxic effects upon cells during culture.
\end{abstract}

Keyword: Medicinal plants; Antioxidant activity; Nitric oxide inhibition 